\title{
Psychological functioning before and after treatment of torticollis with botulinum toxin
}

\author{
Marjan Jahanshahi, C David Marsden
}

\begin{abstract}
The impact of botulinum toxin injection on psychological function was assessed in 26 patients with idiopathic torticollis. Eighty five per cent of the patients and $88 \%$ of the relatives considered torticollis to be better following the injections. Symptomatic improvement with the injections was associated with significant reduction of depression and disability, but non-significant improvement in body concept, and self-esteem. This concordant pattern of change in symptoms and psychological function with the injections supports the proposal that in torticollis depression and disability are consquences of the postural abnormality of the head.
\end{abstract}

In neurological disorders such as spasmodic torticollis, depression can be either secondary to the experience of living with the illness or result from the primary central neurotransmitter dysfunction. The results of a number of previous studies $^{1-4}$ suggested that depression may constitute a reaction to the postural abnormality of the head. Patients with torticollis were found to be more depressed than an equally chronic group of cervical spondylosis sufferers. ${ }^{1}$ Depression in torticollis focussed on a negative view of the self and disfigurement accounted for $14 \%$ of its variance. ${ }^{2}$ In a sample of 67 torticollis patients, a host of psychosocial variables, namely, self-depreciation, disability, degree of control over head position, extent of satisfaction with available social support, and maladaptive coping accounted for $75 \%$ of the variance in depression. In addition, $59 \%$ of the variance of self-depreciation, which was the major predictor of depression, was accounted by the negative body concept resulting from the postural abnormality. ${ }^{3}$ Longitudinal follow up of the patients over 2.5 years revealed that change in the clinical status of torticollis (improved, unchanged, deteriorated) had a significant effect on depression, disability, and body concept. ${ }^{4}$

The above studies provide fairly strong support for the proposal that experience of depression in a proportion of torticollis patients constitutes a reaction to the disorder. A further approach to addressing this issue would be to assess the changes in psychological function before and after a treatment, such as botulinum toxin injections, that successfully controls head deviation. Recently, injections of botulinum toxin into the superficial neck muscles have become the treatment of choice for torticollis, and its efficacy has been evaluated in a number of studies. ${ }^{5-8}$ In most studies, ${ }^{5-7}$ substantial normalisation of head posture with toxin injections was achieved. Despite the popularity and success of botulinum toxin, no study has examined the psychological changes that may be associated with the symptomatic improvement resulting from the injections. The aim of this study was to undertake such an assessment. The specific question addressed was: is improvement of torticollis with botulinum toxin injection .accompanied by improvement of depression, reduction of disability, and improvement of the negative body concept and low self-esteem?

\section{Method \\ Subject}

The sample consisted of 26 cases ( 5 male, 21 female) with a clinical diagnosis of idiopathic torticollis according to previously defined criteria. ${ }^{9}$ Rotational turning or lateral tilting of the head were the major abnormal posture in respectively $10(38.5 \%)$ and four (15.4\%) patients. Forward flexion and backward extension of the head were respectively present in two $(7 \cdot 7 \%)$ and five $(19.2 \%)$ cases. In a further five $(19 \cdot 2 \%)$ cases, the abnormal head posture was a combination of turn/tilt and flexion or extension of the head. The sample was obtained from two sources. Eleven of the patients were all cases who had received botulinum toxin injections after participating in a previous study about two years before. ${ }^{34}$ The other 15 patients were successive first time referrals to a Botulinum Toxin Clinic at the National Hospital for Neurology and Neurosurgery. The mean age of the sample was 51.5 years $(S D=10 \cdot 8)$. The mean age of onset of torticollis was $44 \cdot 3$ years $(S D=10 \cdot 4$, range 24 to 72 years), resulting in an average duration of illness of $7 \cdot 2$ years $(S D=6 \cdot 6$, median $=5.3$ years). All patients had undergone extensive pharmacotherapy including treatment with high-dose anticholinergics which had not been fully successful in controlling their torticollis.

\section{Material}

The following questionnaires were completed by the patients before starting toxin treatment and after one or more such injections. Follow up questionnaires were sent to patients after a minimum interval of three months. 
1) The Beck Depression Inventory $\left(B D I,{ }^{10}\right)$

The 21 items cover cognitive as well as somatic and behavioural symptoms of depression. Patients indicate how they have generally felt during the previous week by selecting one of the four statements for each item. Total scores range from 0 to 63 .

2) The Functional Disability Questionnaire (FDQ)

A 27 item scale was developed to measure disability in daily functioning. Each item is rated on a four point scale (1: "not at all affected", 4: "severely affected"). Details of the FDQ items and data showing it to have high reliability and validity are presented in a previous publicaton. ${ }^{2}$

3) The Body Concept Scale (BCS)

The BCS consisted of 22 pairs of opposite body-related adjectives rated on seven point semantic differential scales. "My body" was the concept rated by the patients. The scores range from 22 to 154 . Details of the BCS and data showing it to be reliable and valid are provided elsewhere. $^{2}$

4) Rosenberg's Self-esteem Scale

The 10 items are rated on a four point agreedisagree format and assess an individual's feelings of self-worth and self-depreciation. Evidence $^{12}$ suggests that the positively and negatively worded items of this scale reflect two independent aspects of self-esteem.

5) Botulinum Toxin Questionnaire

At follow up, the patients indicated the changes in their torticollis with the injections by selecting one of eight categories (much worse, worse, slightly worse, unchanged, slightly better, better, much better, no head deviation). The most important benefits of the injections were indicated by rank ordering the following four statements: "Relief of neck pain", "Straightening of head", "Reduction/cessation of involuntary head movements", "Improved ability to move head in different directions". The patients also described any undesirable side effects and provided the following toxinrelated information: the number of injections, date of the first and last injections, interval between injections, delay in benefit after injections, duration of benefit.

6) Rating of toxin-related change by a relative

At follow up, a spouse or relative indicated how the patient's torticollis compared before the injections using the same scale as the patients.

Statistical Analysis: Where the data were derived from interval scales and were normally distributed, parametric tests (paired $t$ test) were used. The non-parametric Mann-Whitney $U$ test was used when the number of cases was small.

\section{Results \\ 1 Change in torticollis with botulinum toxin injection}

The mean time since the first injection was 29.9 weeks $(S D=26 \cdot 2)$, with a range of eight weeks to 21 months. The mean number of injections was three, with a range of one to 11 injections. The mean delay in the onset of benefit was 8.9 days (median five days, range one to 28 days). The average duration of benefit was eight weeks $(S D=3 \cdot 2)$ with a minimum of one and a maximum duration of benefit of 14 weeks. The average interval between successive sets of injections was 11.6 weeks $(\mathrm{SD}=2 \cdot 0)$.

Twenty two of the 26 patients (84.6\%) considered their torticollis to be better as a result of the injections. Torticollis was reported to be unchanged in two $(7 \cdot 7 \%)$ and worse in two patients. $(7 \cdot 7 \%)$ following the injections compared before such treatment. The ratings of change by the relatives were similar; with improvement, no change and deterioration respectively reported in $88 \%, 8 \%$, and $4 \%$ of the patients.

Straightening of the head was rank ordered as the first or second most important benefit of the injections by 14 patients. Relief of neck pain was rank ordered as the first or second most important benefit by 11 cases. Nine patients considered reduction of involuntary movements of the head as the first or second most beneficial result. Improved ability to move the head in different directions was ranked as the first or second most important benefit derived from the injections by seven patients.

In eight cases the injections reportedly produced no side effects. Feelings of stiffness in the neck or floppiness of the head were reported as side effects by 10 patients $(38.5 \%)$. Difficulty swallowing and lack of energy were respectively the second and third most common side effects reported by seven $(26.9 \%)$ and six (23\%). Five patients $(19 \cdot 2 \%)$ reported that their voice or speech was in some way affected.

\section{Changes in psychological function with}

botulinum toxin

There were no male/female differences on any of the pre- or post-toxin measures of psychological function $(p>0.05)$. Two questions are relevant regarding the changes in psychological function associated with the injections. First, are different types of change (improved versus unimproved) in the postural abnormality of the head with the injections associated with differential change in psychological function? This question is addressed by comparing the changes in psychological function for the 22 improved and four unimproved cases. The second question is whether improvement in torticollis is associated with significant improvement in psychological function. This question was addressed by comparing the scores on the psychological measures before and after the injections for the 22 improved cases.

To provide a picture of the differences in the psychological effects of the injections for improved and unimproved cases, the 22 cases whose torticollis was improved were compared with the four cases whose head deviation had not benefited or had deteriorated after the injections. There were no differences in the pre-treatment scores of the improved and unimproved cases on any measure of psychological function $(p>0.05)$. However, the four patients who had not benefited from the 
Table Mean (SD) scores of the 22 improved cases before and after treatment with botulinum toxin injections

\begin{tabular}{lll}
\hline & Before & After \\
\hline Depression & $14.4(10.9)$ & $11.3(9.9)$ \\
Disability & $52.9(14.6)$ & $46.4(15.7)$ \\
Body concept & $79.5(28.9)$ & $69.2(30.7)$ \\
Self-esteem & & \\
$\quad$ Self-worth & $13.3(3.4)$ & $14.5(2.6)$ \\
Self-depreciation & $14.6(3.4)$ & $13.9(3.8)$ \\
\hline
\end{tabular}

injections showed less improvement in depression (Mann-Whitney $z=2.3, p=0.02$ ) and body concept (Mann-Whitney $z=2 \cdot 2$, $\mathrm{p}=0.02$ ) than those whose torticollis had improved. There were no differences between the improved $(n=22)$ and unimproved $(n=4)$ cases in terms of disability, self-worth, and self-depreciation ( $p>0.05$ ).

Given the significant differences between the improved and unimproved subgroups in depression and body concept, inclusion of the four cases who noted their torticollis to be unaltered or worse following the injections, may mask or dampen the beneficial effects of the injections on psychological function, by rendering the sample less homogeneous. These four cases were therefore excluded before examining whether improvement of torticollis was associated with improved psychological function from before to after the injections. For the remaining 22 cases, the mean and standard deviation scores on the measures of psychological function before and after treatment with botulinum toxin are shown in the table. There was a significant improvement in depression ( $t=2 \cdot 7, \mathrm{df}=21, \mathrm{p}=0.02)$, and disability $(t=2 \cdot 6, d f=21, p=0.02)$. The improvement in body concept was not significant $(t=1.8, d f=18, p=0.08)$. Selfworth and self-depreciation were also not significantly altered from before to after the injections $(p>0.05)$.

\section{Discussion}

Botulinum toxin injection was an effective treatment for the majority of cases. Improvement was reported by $85 \%$ of patients and $88 \%$ of the relatives. Straightening of the head and relief of neck pain were the two most common reported benefits of the injections. Stiffness or floppiness of the head and difficulty swallowing were the most common side effects. The effects of botulinum toxin in the present sample were in all respects comparable to those reported in previous studies. ${ }^{5-8}$

Reduction of depression and disability were the major psychological benefits of the injections. After the injections, body concept had shifted towards a more positive view of the body, although the changes were not significant. The beneficial effects of the injections wear off as the time interval between the injections increases and for most patients the head deviation and involuntary movements return during the period between successive injections. Therefore, as freedom from the postural abnormality of the head is neither complete nor permanent, it is feasible that alterations of body concept towards the positive end of the scale may also never be complete with this form of symptomatic treatment.
The concurrent improvement of depression and disability with successful treatment of torticollis with botulinum toxin injections (or as a non-specific result of undergoing such treatment) provides support for the reactive nature of depression and disability present in a proportion of torticollis patients and confirm the findings of previous studes. ${ }^{1-4}$ The four cases who had not benefited from the injections showed less improvement in depression and body concept after the injections compared with the majority who had benefited, and provides further supportive evidence for the reactive model.

What are the implications of these findings for the management of torticollis? The results showed that successful treatment of torticollis with botulinum toxin injections was accompanied with improvement in depression and disability. Existing evidence ${ }^{5-8}$ suggests that botulinum toxin injections are currently the most successful symptomatic treatment of torticollis. Our results provide further and more wide ranging evidence in support of the value of the injections. However, two aspects of psychological function, negative body concept and low self-esteem, were not altered significantly with the symptomatic improvement of the postural abnormality of the head. This resistance to change in body concept and selfesteem may relate to the fact that normalisation of the head deviation with the injections is neither complete nor permanent and the postural abnormality may recur in the interval between injections. Alternatively, it is feasible that alterations of body concept and low selfesteem may have become partially dissociated from the postural abnormality and maintained by other independent factors. Use of cognitivebehavioural techniques for more direct management of these aspects of psychological function, such as, negative body concept and low self-esteem, may also be appropriate.

This work was supported by a grant from the Medical Research Council.

1 Jahanshahi M, Marsden CD. Depression in torticollis: A controlled study. Psychol Med 1988;18:925-33.

2 Jahanshahi $M$, Marsden $C D$. Body concept, depression and disability in spasmodic torticollis. Behavioural Neurol 1990;3:117-31.

3 Jahanshahi M. Psychosocial factors and depression in torticollis. F Psychosomatic Res 1991b;35:493-507.

4 Jahanshahi M, Marsden CD. A longitudinal study of depression, disability and body concept in torticollis. Behavioural Neurol 1990;3:233-46.

5 Tsui JK, Eisen A, Stoessl AJ, Calne S, Calne DB. Doubleblind study of botulinum toxin in spasmodic torticollis. Lancet 1986;245-7.

6 Brin MF, Fahn S, Moskowitz C, et al. Localised injections of botulinum toxin for the treatment of focal dystonis and hemifacial spasm. In: Brin MF, Fahn S, Moskowitz C, hemifal spasm. In. Brin MF, Fahn S, Moskowiz C, et 1988:599-608.

7 Stell R, Thompson PD, Marsden CD. Botulinum toxin in spasmodic torticollis. $\mathcal{f}$ Neurol Neurosurg Psychiatry spasmodic tor $1988 ; 51: 920-3$.

8 Gelb DJ, Lowenstein DH, Aminoff MJ. Controlled trial of botulinum toxin injections in the treatment of spasmodic botulinum toxin injections in the treat
torticollis. Neurology 1989;39:80-4.

9 Barbeau A, Calne DB, Fahn S, et al. Classification and investigation of dystonia. In Marsden CD, Fahn S, eds. Movement Disorders II, London: Butterworths, 1987 332-58.

10 Beck AT, Ward CH, Mendelson M, Mock JE, Erbaugh JK. An inventory for measuring depression. Arch Gen Psychiatry 1961;4:561-71.

11 Rosenberg MI. Society and the adolescent self-image. Princeton: Princeton University Press, 1965.

12 Parry G, Brewin CR. Cognitive style and depression: Symptom-related, event-related or independent provoking factor? Br $\mathcal{F}$ Clin Psychol 1988;27:23-35. 